\title{
Lattice Thermal Conductivity of Multi-Component Alloys
}

\author{
M. Caro $^{1}{ }^{*}$ L. K. Béland ${ }^{2}$, G. D. Samolyuk ${ }^{2}$, R. E. Stoller ${ }^{2}$, and A. Caro ${ }^{1}$ \\ 1-Materials Science and Technology Division, Los Alamos National Laboratory, Los Alamos, NM 87545 and \\ 2-Materials Science and Technology Division, Oak Ridge National Laboratory, Oak Ridge, TN 54321
}

\begin{abstract}
High entropy alloys (HEA) have unique properties including the potential to be radiation tolerant. These materials with extreme disorder could resist damage because disorder, stabilized by entropy, is the equilibrium thermodynamic state. Disorder also reduces electron and phonon conductivity keeping the damage energy longer at the deposition locations, eventually favoring defect recombination. In the short time-scales related to thermal spikes induced by collision cascades, phonons become the relevant energy carrier. In this work, we perform a systematic study of phonon thermal conductivity in multiple component solid solutions represented by Lennard-Jones (LJ) potentials. We explore the conditions that minimize phonon mean free path via extreme alloy complexity, by varying the composition and the elements (differing in mass, atomic radii, and cohesive energy). We show that alloy complexity can be tailored to modify the scattering mechanisms that control energy transport in the phonon subsystem. Our analysis provides a qualitative guidance for the selection criteria used in the design of HEA alloys with low phonon thermal conductivity.
\end{abstract}

Keywords: High entropy alloys, thermal transport, thermal properties, radiation resistance, Molecular Dynamics simulations

In the search for new materials with outstanding properties for novel applications, a new direction involving high entropy alloys (HEA) is proving promising. These materials composed of more than five elements, have shown improved strength, and remarkable fracture toughness such as in the case of CrMnFeCoNi alloy [1] with toughness levels comparable to Ni-based super alloys and several stainless steel such as 316 SS. By a mixture method of synthesis, design, and element selection, high entropy alloys have been developed for advanced applications, e.g. $\mathrm{Co}_{1.5} \mathrm{CrFeNi}_{1.5} \mathrm{Ti}_{0.5}$ with high temperature strength and oxidation resistance, which is proposed for high temperature tensile testing machines up to $1000^{\circ} \mathrm{C}$, or $\mathrm{Al}_{0.3} \mathrm{CrFe}_{1.5} \mathrm{MnNi}_{0.5}$ with high hardness and wear resistance which could replace shot peening or other methods currently used for surface hardening [2]. Also, single-phase solid solution HEAs have been investigated such as FCC CoCrFeNi [3] and CoCrFeMnNi [4] and BCC alloys such as WNbMoTa, WNbMoTaV, and TaNbHfZrTi [5].

High entropy of mixing is the main characteristics of HEA alloys, i.e. high configurational entropy due to the large number of elements at, or close to, equiatomic composition. The combination of different elements in HEA alloys, including elements with large atomic radius difference, may induce lattice distortion [6], which in turn influences the alloy structure and properties.

HEA could have potential applications in radiation environments if extreme disorder improves their damage response. A possible element is their ability to recover damage by increasing defect recombination soon after the damage energy is deposited in the lattice. This effect could be due to a longer duration of the thermal spike produced by a reduced thermal conductivity. Clearly,

*Electronic address: magda@lanl.gov supporting experimental evidence is still missing. To our knowledge, very few experimental studies have been performed on radiation effects in high entropy alloys [7]. A scoping study by Kumar et al. [8] showed poor phase stability for the examined single-phase polycrystalline FCC FeNiMnCr alloy. Significant (Cr and $\mathrm{Mn}$ ) radiation induced segregation at grain boundaries was observed in the alloy specimens after $3 \mathrm{MeV} \mathrm{Ni}$ ion irradiation at $500^{\circ} \mathrm{C}$ to 1 and $10 \mathrm{dpa}$. In contrast, Nagase et al. [9] reported phase stability for $\mathrm{CoCrCuFeNi} \mathrm{HEA}$ after fast $(2$ $\mathrm{MeV}$ ) electron irradiation to over $40 \mathrm{dpa}$ at room temperature $(298 \mathrm{~K})$ and $500^{\circ} \mathrm{C}(773 \mathrm{~K})$. HEA behavior under irradiation still remains vastly unexplored.

At the center of this topic is the hypothesis that HEA inherent high configuration entropy will promote the stability of the solid solution phase and that reduced thermal conductivity will promote defect recombination, enhancing radiation resistance. Defect recombination could be enhanced by increasing the time at which thermal energy remains close to the high-energy collision cascades sites. This thermal energy is transported away by phonons, electrons, and magnons. In kinetic theory of gasses, thermal conductivity depends on the product of the group velocity and carrier mean free path. Mean free path of carriers decreases as a consequence of extreme disorder. Thermal conductivity of metals is primarily due to electrons. Phonons become relevant only in the short time scale related to thermal spikes in collision cascades, where energy is deposited first into the ions, and only after a time scale given by the strength of the electron-phonon interaction, the electrons start capturing and conducting this energy away from the deposition region. Therefore, phonons, although not the main heat carriers in metals under normal conditions, become relevant during the time scale of few picoseconds of the heat spike regime.

HEA thermal conductivity could be tuned by varying the alloy elemental composition and concentration. 
Computer simulations can efficiently help in the search for optimized HEA with desired properties, as we show in this work. Using ab initio molecular dynamics simulations and CALPHAD methodology, Gao et al. identified several possible FCC and HCP multicomponent solid solution compositions in several HEA alloys [3]. Using molecular dynamics with a tight-binding potential, Kao et al. investigated the rapidly solidified structure of equimolar HEAs with eight elements Ni-Al-Cu-Co-Ti-V$\mathrm{Zn}-\mathrm{Zr}$ and explored the effect of the variation in atomic structure and size [10]. An atomistic modeling approach was applied to study W-Nb-Ta-Mo and W-Nb-Ta-Mo-V HEA with refractory elements [11]. First-principle studies within density-functional theory (DFT) have been performed to determine the composition effect on lattice constant and formation enthalpy of the five-element FeNiCrCuCo HEA alloy [12].

In this work, Non-Equilibrium Molecular Dynamics (NEMD) and equilibrium Green-Kubo (GK) approaches are applied to study phonon thermal conductivity of multicomponent alloys, aiming at understanding the role of alloy complexity on thermal transport properties. Our study focuses on the effect that the choice of elements and composition has on reducing HEA thermal conductivity. We perform a systematic study of phonon thermal conductivity, $\kappa$, in multiple component solid solutions represented by LJ potentials, looking for conditions minimizing phonon mean free path via extreme alloy complexity. We find that by varying alloy constituents, i.e. by combining elements in the alloy with different size, mass, and cohesive energy, different phonon thermal conductivities are obtained. We show that alloy complexity can be tailored to modify the scattering mechanisms that control energy transport in the phonon subsystem. Our analysis provides guidance for the design of HEA alloys with low phonon thermal conductivity, which entails maximum phonon scattering.

\section{SIMULATION METHODS}

While strictly speaking HEA are those composed of five or more components, we restrict our study to binaries, ternaries, and quaternaries, where the parameter space is large but manageable, and we call them HEAs. To study the interactions between elements of multicomponent alloys, we use a model system described by the Lennard-Jones (LJ) potential given in Eq. 1:

$$
V_{i j}\left(r_{i j}\right)=4 \epsilon\left[\left(\sigma / r_{i j}\right)^{12}-\left(\sigma / r_{i j}\right)^{6}\right],
$$

$V_{i j}$ in Eq.1 is the potential energy of a pair of particles ( $i$ and $j$ ) separated by a distance $r_{i j}$, parameter $\epsilon$ is the potential well depth, and $\sigma$ is the distance at which $V_{i j}(\sigma)$ $=0$. The equilibrium pair separation is $2^{1 / 6} \sigma$. A switching function with a cut-off distance $\mathrm{r}_{c}=2.5$ is used to have a smooth truncation in the simulations. The ground state is an FCC crystal.
TABLE I: Lennard-Jones $\epsilon$, and $\sigma$ parameters for elements typically present in HEA alloys.

\begin{tabular}{lll}
\hline Element & $\epsilon(\mathrm{eV})$ & $\sigma(\AA)$ \\
\hline $\mathrm{Al}$ & $0.392 \mathrm{eV}$ & 2.620 \\
$\mathrm{Co}$ & $0.516 \mathrm{eV}$ & 2.284 \\
$\mathrm{Cr}$ & $0.502 \mathrm{eV}$ & 2.336 \\
$\mathrm{Cu}$ & $0.409 \mathrm{eV}$ & 2.338 \\
$\mathrm{Fe}$ & $0.527 \mathrm{eV}$ & 2.321 \\
$\mathrm{Ni}$ & $0.520 \mathrm{eV}$ & 2.282 \\
$\mathrm{Si}$ & $0.0175 \mathrm{eV}$ & 3.826 \\
$\mathrm{Zr}$ & $0.739 \mathrm{eV}$ & 2.932 \\
\hline
\end{tabular}

In this study, we vary the HEA constituent properties, i.e. elements size, and cohesive energy, by varying $\sigma$ and $\epsilon$ parameters in Eq. 1. The range in $\sigma$ and $\epsilon$ is selected to adequately represent several typical elements in HEA, such as those listed in Table 1, which allows a one-toone relation between the model LJ system and some real crystal.

To analyze the effect of element mass, atomic size, and cohesive energy on the thermal conductivity, $\kappa$, of the LJ solid, NEMD and GK simulations were performed. The NEMD technique is known to reproduce well phononic transport properties $[13,14]$. In the direct thermostatting method [15], a heat flux is applied across the $\mathrm{z}$ axis of a rectangular box and a steady state, one-dimensional temperature gradient is established. The flux of energy, $\Delta Q$, needed to maintain the temperature gradient, $\Delta T$, is monitored during the simulation run. In this case, the thermal conductivity, $\kappa$, is given in Eq. 2:

$$
\kappa=\Delta Q \Delta z /(2 A \Delta t \Delta T)
$$

where $\Delta z$ is the box height, $A$ is the cross-sectional area of the box, and $\Delta t$ is the time step in the simulation.

A second approach to compute thermal conductivity of a solid makes use of the equilibrium method based on Green-Kubo (GK) formalism [16]. In this case, the transport coefficient $\kappa$ is given in terms of the integral over time $t$, of the ensemble average of the auto-correlation of the heat flux, J, given in Eq. 3:

$$
\kappa=V /\left(3 k_{B} T^{2}\right) \int_{0}^{\infty}<\mathbf{J}(0) \mathbf{J}(t)>d t
$$

where $V$ is the box volume, $T$ is the solid temperature, and $k_{B}$ is the Boltzmann constant. The local heat flux, J, given by Eq. 4, is calculated by computing the per-atom energy (kinetic and potential), $e_{i}$, the atomic velocity, $\mathbf{v}_{i}$, and per-atom stress, $\mathbf{S}_{i}$.

$$
\mathbf{J}=\frac{1}{V}\left[\sum_{i} e_{i} \mathbf{v}_{i}-\sum_{i} \mathbf{S}_{i} \mathbf{v}_{i}\right]
$$




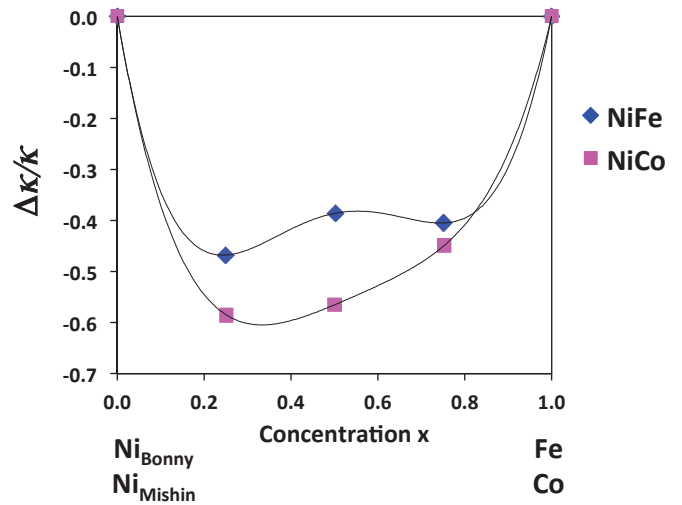

FIG. 1: Relative change in thermal conductivity, $\Delta \kappa / \kappa$, in FCC binary alloys as a function of components concentration (NiFe - diamonds, and NiCo - squares)

\section{RADIATION DAMAGE SIMULATIONS}

To place our research in context, we study first the effects of thermal conductivity on the duration of thermal spikes induced by collision cascades. We aim at proving that by varying thermal conductivity we can affect the duration of the spike. The actual proof that by varying thermal conductivity we can alter the defects production, is beyond the scope of this work and will be reported in a forthcoming publication.

To study collision cascades and defect formation, reliable interatomic potentials are desirable, as those commonly called many-body potentials, which are well beyond the simplicity of the LJ model. At present however, embedded atom model (EAM) potentials are not readily available for multicomponent systems. For this reason, and to investigate radiation effects on HEA alloys, MD simulations of radiation damage were carried-out on binary NiFe and NiCo alloys, i.e. two typical HEA elements for which EAM models for the pure elements and their mixtures exist. The thermal conductivity was calculated using GK approach, see [17] for details.

As shown in Fig. 1, thermal conductivity in NiFe alloy is reduced from $8 \mathrm{~W} / \mathrm{mK}$ for pure $\mathrm{Ni}$ to $4 \mathrm{~W} / \mathrm{mK}$ with introduction of Fe substitution atoms, and then is restored to $6 \mathrm{~W} / \mathrm{mK}$ in pure FCC Fe. The cause of the thermal conductivity reduction is the disorder introduced by both the difference in mass between $\mathrm{Ni}$ and $\mathrm{Fe}$, and the difference in force constants because they are different elements, i.e. diagonal and off-diagonal disorder in the dynamical matrix describing phonons. In NiCo alloy, the masses of both components are very similar, and the disorder is introduced by the different values of force constants. In this case, thermal conductivity values drop significantly even at small Co concentrations.

Simulations of $1 \mathrm{keV} \mathrm{Ni}$ cascades in equiatomic

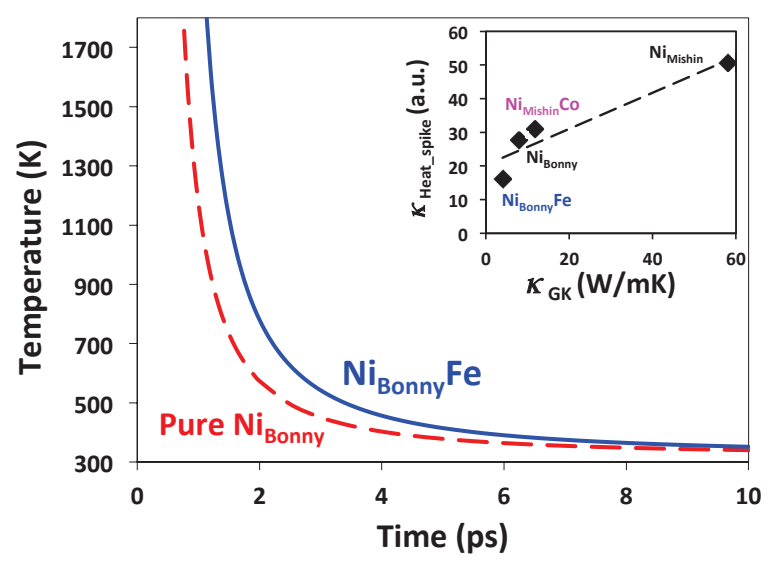

FIG. 2: Main panel) Temperature at the core of a $1 \mathrm{keV}$ displacement cascade, calculated using EAM interatomic potentials for $\mathrm{NiFe}$ (solid), and $\mathrm{Ni}_{\text {Bonny }}$ (dashed). Inset) Relation between the conductivity determined by fitting the thermal spike with a heat equation assuming a Gaussian temperature profile, and the value determined using GK formalism.

$\mathrm{NiFe}$ and NiCo solid solutions and the pure elements were performed using LAMMPS (Large-scale Atomic/Molecular Massively Parallel Simulator, see http://lammps.sandia.gov) [18] and EAM NiFe potential by [19], and NiCo potential obtained by crossing EAM interatomic potentials for pure $\mathrm{Ni}$ by [20] and pure $\mathrm{Co}$ by [21]. The time evolution of the temperature in the region of the cascade core was monitored, and results for alloys and pure elements were compared. Fig. 2 main panel clearly shows that the cascade-core temperature is much larger in $\mathrm{NiFe}$ than in pure $\mathrm{Ni}$, implying that there is a decrease in the rate at which energy is transported away from the cascade-core deposition region. Results for NiCo show a similar but less marked behavior.

In Fig. 2 inset, we plot the thermal conductivity obtained from a fit of the temperature evolution given in Fig. 2 main panel, to a spherically symmetric heat diffusion equation, to obtain $\kappa_{\text {Heat-spike, versus the thermal }}$ conductivity of the same systems calculated using GK approximation, $\kappa_{G K}$. The strong correlation between these variables, although not perfect, provides a strong evidence that the duration of heat spikes is, to a large extent, controlled by lattice thermal conductivity.

\section{NEMD AND GK SIMULATIONS}

Simulations were performed using LAMMPS [18]. The crystal structure in the simulations is face-centered-cubic (FCC) with lattice constant relaxed to its equilibrium value for each case. The simulations are performed in dimensionless LJ units with LJ reduced units defined as $E^{*}=E / \epsilon$ for energy, $t^{*}=t\left(\epsilon / m / \sigma^{2}\right)^{1 / 2}$ for time, $T^{*}$ 
$=T k_{B} / \epsilon$ for temperature, and $x^{*}=x / \sigma$ for distance. Different masses are considered during the simulation, with mass, $m$, in arbitrary units. In NEMD simulations, a rectangular box containing 72000 atoms was used, with hot regions in both borders of the solid, and a cold region at the center. Periodic boundary conditions are used in all directions. All simulations use a time step $\Delta \mathrm{t}=0.005$.

After a conjugate gradient (CG) minimization, atoms in the box are first equilibrated to a temperature $T^{*}=$ 0.2 (equivalent to about $1 / 3$ of the melting temperature) for 5000 steps. Simulations are run in the NVT (constant mass, volume, and temperature) ensemble, at zero pressure. A temperature gradient is then established in a second equilibration run for 10000 steps. Hot regions in the temperature profile are set to a temperature $T^{*}$ hot $=0.25$, and the central cold region to $T^{*}$ cold $=0.15$. A Langevin thermostat is applied to the group of atoms in the hot, and cold regions, separately. Averages over 20000 steps are used to compute the flux of energy, $\Delta Q$, as well as to determine the temperature gradient, $\Delta T$.

Thermal conductivity is also determined using the Green-Kubo (GK) methodology. In GK simulations, a cubic box containing 32000 atoms is used. Simulations start with a CG minimization, followed by a thermal equilibration at temperature $T^{*}=0.2$ for 5000 steps, also using a time step equal to 0.005. The dynamics of the system is followed over long time scales, with heat flux values determined at each time step. The data is used to compute the heat flux autocorrelation across varying delay times. To ensure convergence of the correlation function, thermal conductivity data are obtained after 3 million steps, in averages that use a sampling interval $\mathrm{s}$ $=2$ time steps, and a correlation length $\mathrm{p}=300$ time steps. Using these parameters, a proper sampling is obtained, allowing the system the time needed for the heat flux fluctuations around zero to dissipate.

\section{THERMAL CONDUCTIVITY IN A BINARY ALLOY}

We first investigate the thermal conductivity of binary solids at equiatomic composition. Three cases were analyzed using GK simulations, a) an alloy composed of atoms $A$ and $B$, differing in mass, $m$ and atomic size, $\sigma$, b) an alloy with equal atomic size but differing in mass $m$ and cohesive energy $\epsilon$, and c) an alloy with equal masses but differing in atomic size $\sigma$, and cohesive energy $\epsilon$.

Fig. 3 displays 2D maps of the phonon thermal conductivities as calculated. Frontiers between colors are iso-conductivity lines. In the first case, shown in Fig. 3a, a decrease in $\kappa$ values is observed as the difference in mass and atomic size increases. A significant drop in conductivity is observed, namely a factor of 4 for $16 \%$ change on atoms size, i.e. large atom radius is 1.08 and small radius is 0.92 . Also, $\kappa$ drops by a factor of 3.4 for a factor of 3 in the mass difference, i.e. heavy atom mass 1.5, and light atom mass 0.5. The center of the figure represents a unary system, since both atoms are identical, with maximum $\kappa$ value. The figure has inversion symmetry, i. e. first and third quadrants are equal, as are second and fourth, since they correspond to changing the labels $A$ and $B$. The most interesting feature of this figure is the fact that the iso-conductivity lines are tilted, suggesting that the two scattering mechanisms, namely mass defect and size defect, do not add independently; instead, there is a larger drop in conductivity if the heaviest atom is the smallest.

The stars in Fig. 3a mark the approximate location of the binary systems NiCo, NiFe, and NiPd [22].

Fig. $3 \mathrm{~b}$ shows the $2 \mathrm{D}$ representation of $\kappa$ iso-lines as a function of the difference between the atomic size, $\sigma$, and cohesive energy $\epsilon$. In this case, and for the range of parameters explored, a small coupling between mass and force-constant defects is observed. A similar behavior is observed in Fig. 3c, where $\kappa$ values are reported as a function of atomic size, $\sigma$, and cohesive energy $\epsilon$ difference between alloy constituents.

\section{THERMAL CONDUCTIVITY OF A MULTI-COMPONENT ALLOY}

Having determined in the previous section that a combination of mass and size is the most efficient way to reduce the thermal conductivity, in this section we analyze equiatomic quaternary alloys composed of elements obtained by combination of 4 possible masses $(m=0.7$, $0.85,1.15,1.30)$, and 4 possible radii $(\sigma=0.92,0.96$, 1.04 , and 1.08). There are 24 possible quaternary "alloys" in our ad-hoc sand box. We calculated the thermal conductivity of all of them, and in Fig. 4 we display the six cases with lowest and the six cases with highest conductivity.

To identify the elements in the figure, those with increasing $\sigma$ are represented by trapezoids increasing in size, while their mass is represented by a color map, with yellow, green, blue, and black colors indicating elements with increasing mass, respectively. The results indicate that a variation of up to $18 \%$ between the maximum and minimum values of conductivity can be obtained if the heavier elements are at the same time the smaller. It is also interesting to note that the reciprocal behavior is observed, namely a combination of lighter elements and smaller atomic radii gives an alloy with the larger values of $\kappa$.

Having determined the influence of atomic properties on thermal conductivity, we select now one particular alloy, namely the third from the left in Fig. 4 to study the influence of composition on thermal conductivity. We choose this alloy (although not the one with minimum conductivity) because its elements, that we call $A, B, C$, and $D$, are arranged in a simple way: the largest is the lightest, $A$, the smallest is the heaviest, $D$, and similarly for the two other.

We report $\kappa$ values as a function of element concentra- 


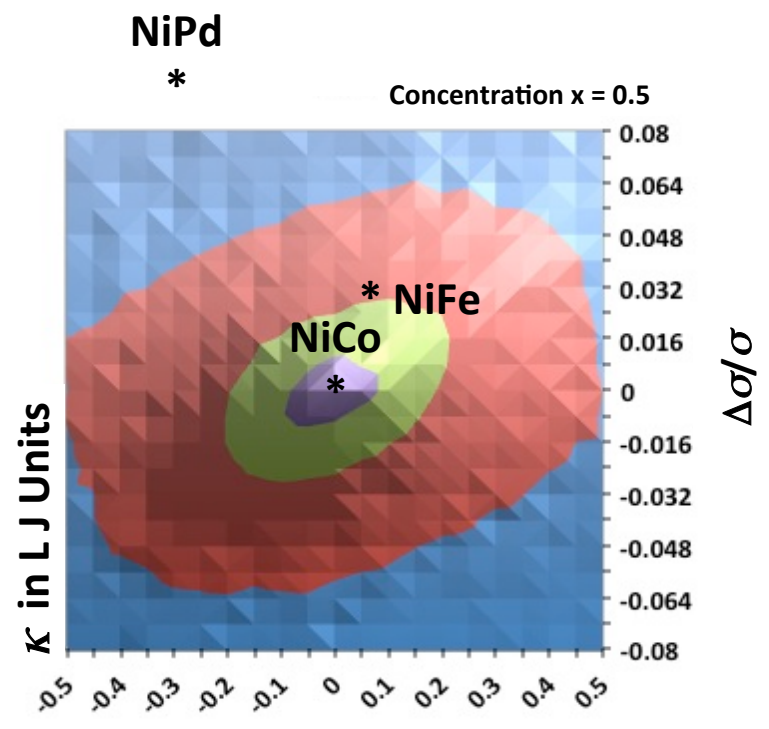

\section{$\Delta m / m$}
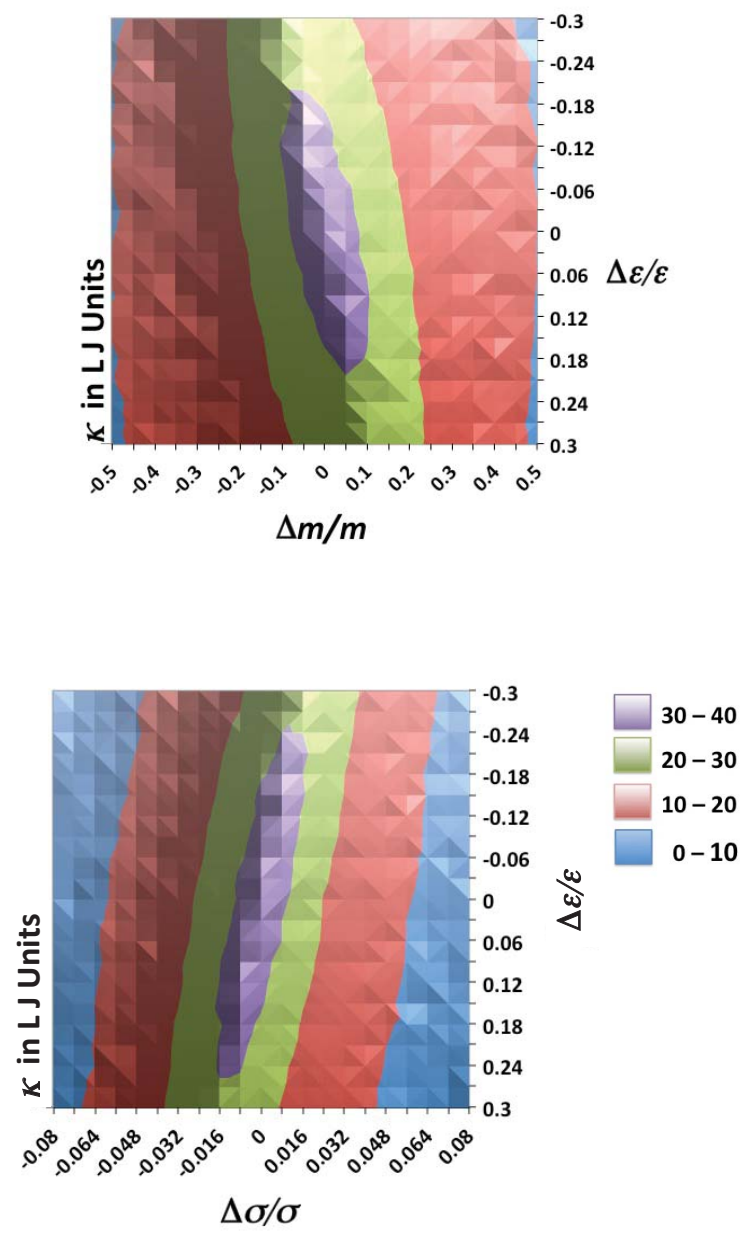

FIG. 3: Thermal conductivity $\kappa$ for a binary solid at equiatomic composition a) difference in mass $m$, and atomic size $\sigma, \mathrm{b}$ ) difference in mass $m$, and cohesive energy $\epsilon$, and c) difference in atomic size $\sigma$, and cohesive energy $\epsilon$.

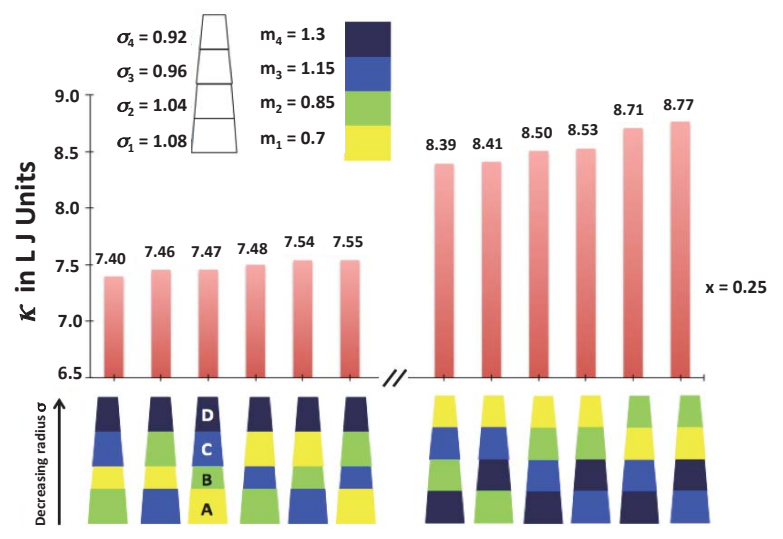

FIG. 4: Thermal conductivity $\kappa$ of a LJ alloy composed of four elements at equiatomic composition. Y-axis: $\kappa$ values for 12 cases out of 24 possible combinations. X-axis: Combination of $m$, and $\sigma$ values for each case. Upper left corner: ColorSymbol map for elements $A, B, C$, and $D$, where trapezoids of increasing size, represent elements with increasing atomic size, $\sigma$, and colors yellow, green, blue, and black, represent elements with increasing mass values, $m$.

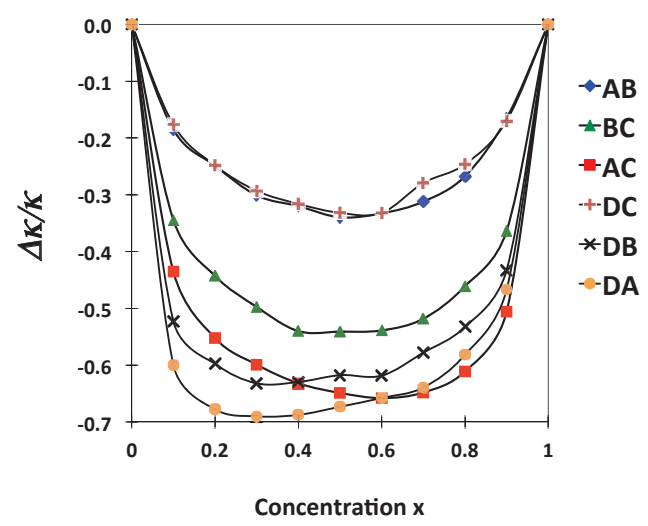

FIG. 5: Thermal conductivity $\kappa$ for the case of elements $A$, $B, C$, and $D$, increasing in mass $m$, and decreasing in radius $\sigma$. $\kappa$ values for binaries $A B$ (diamonds), $B C$ (triangles), $A C$ (squares), $D C$ (roman cross + ), $D B$ (cross $\mathrm{x}$ ), and $D A$ (circle).

tion for the edges (quasi-binaries), for the sides (quasiternaries) and for the bulk (quaternary) tetrahedron of composition. Six cases correspond to the sides of a tetrahedron. Fig. 5 displays $\kappa$ values obtained for binary systems with components $A B, B C, A C, D C, D B$, and $D A$, respectively. Minimum $\kappa$ values are obtained for case $D A$, corresponding to an alloy in which the heavier atom is the smallest, i.e. atom $D$, and the light atom is the largest, i.e. atom $A$. Note the curvature in Fig. 


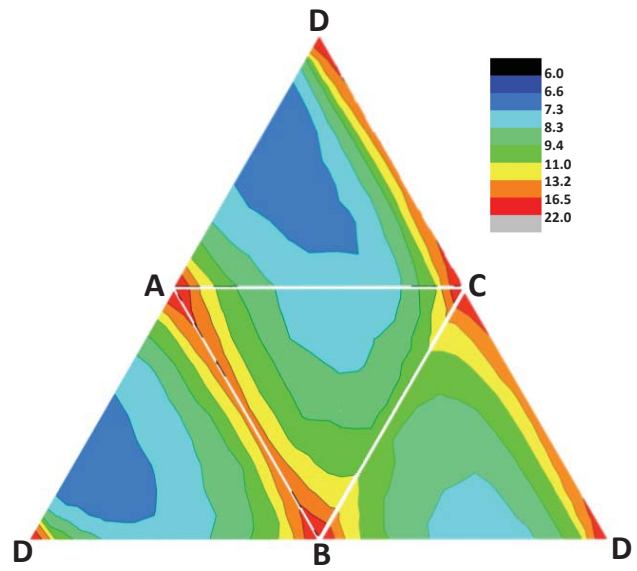

FIG. 6: 2D cuts of a quaternary composition diagram indicating the location of the minimum conductivity in terms of composition.

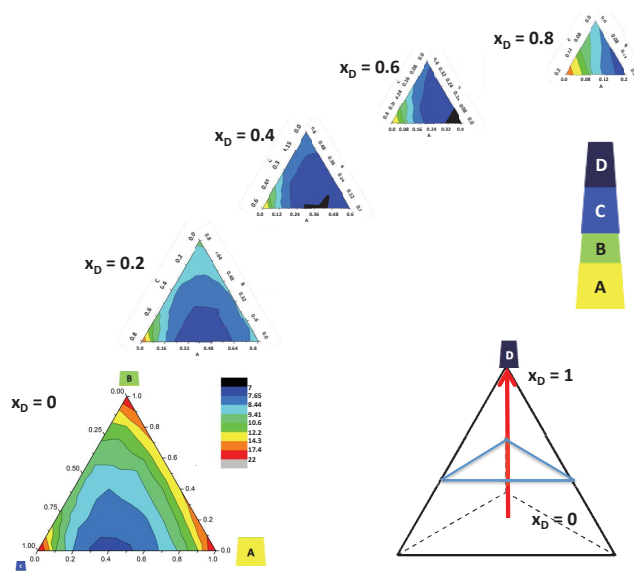

FIG. 7: 2D cuts of a quaternary composition diagram in z-axis direction; black regions indicate the location of the minimum conductivity in terms of composition.

5 case $D A$ at concentration $\mathrm{x}_{A}=0.3$, indicating that larger concentrations of element $D$ favors slightly lower $\kappa$ values.

For the four quasi-ternaries, Fig. 6 shows the isoconductivity lines. It is clearly seen here, that the binary $A D$ has a strong influence in defining low conductivity compositions, where the incorporation of some $B$ or some $C$ decreases it even further. Finally, Fig. 7 shows the bulk of the tetrahedron represented by cuts at constant $D$-composition. We see that the side defined by $A C D$ displays the darkest color (black) of the lowest possible conductivity. Inspection of the data reveals that the global minimum is found for the ternary alloy $A_{0.4} C_{0.1} D_{0.5}$.

\section{DISCUSSION}

Mass, size and force constant defects are the three parameters that characterize defects in the harmonic
Hamiltonian that represents phonon thermal conductivity. In a disordered alloy, they may appear individually or collectively, depending on the constituent atoms. In multicomponent alloys it is expected that all three mechanisms will contribute to affect thermal conductivity. It is common in the description of scattering processes to assume that each scattering mechanisms acts independently from each other [23]; under this approximation, an expression for the total mean free path of the carriers, or for the relaxation time is,

$$
\frac{1}{\tau_{\text {total }}}=\frac{1}{\tau_{\text {mass }}}+\frac{1}{\tau_{\text {size }}}+\frac{1}{\tau_{\text {force-constant }}}
$$

Our simple calculations show that this approximation is not adequate for multicomponent alloys of relevance in the field of HEA. Coupling between the disorder sources is not negligible, in particular for mass and size defects, and to a less extend for the other combinations, as shown in Fig. 3. This observation suggests that if there is some freedom in the choice of elements in a particular HEA, as the parameter space for these alloys is very large, particular combinations of size, mass, and cohesive energy may produce alloys with tunable thermal conductivity, while keeping other properties, such as configurational entropy unchanged.

The influence of phonon thermal conductivity on the life-time of the thermal phase of a collision cascade is quite understood, as was presented here in Fig. 2, which can be summarized as: a change in thermal conductivity by a factor $\alpha$ implies a change in quenching time by a factor $1 / \alpha$. Our results then suggest that it is possible to tune the life-time of cascades by a judicious choice of elements in a multicomponent alloy.

The relation between life-time of collision cascades and defect production is a more difficult question that we do not address in this work. However, we speculate that radiation resistance could be improved by optimizing phonon scattering / thermal conductivity via a proper choice of elements composing the HEA, and composition.

In this work, we determined the best composition for multicomponent alloys with minimum $\kappa$ using LJ representative models of alloys. Our MD simulations show that different disorder sources or scattering mechanisms do not add up independently, but show significant coupling, in particular for size and mass defects.

\section{Acknowledgments}

Fruitful discussions with R. B. Schwarz are gratefully acknowledged. Work supported by the Energy Dissipation to Defect Evolution Center (EDDE), an Energy Frontier Research Center funded by the U.S. Department of Energy, Office of Science. 
[1] B. Gludovatz, A. Hohenwarter, D. Catoor, E.H. Chang, E.P. George, R.O. Ritchie, Science 345, 6201 (2014) 11531158.

[2] B.S. Murty, J.W. Yeh, S. Ranganathan, "HighEntropy Alloys", 1st Ed. Butterworth-Heinemann Editors, (2014), pp. 218.

[3] M.C. Gao, and D.E. Alman, Entropy 15 (2013) 45044519.

[4] F. Otto, Y. Yang, H. Bei. E.P. George, Acta Mater. 61 (2013) 2628-2638.

5] O.N. Senkov, J.M. Scott, S.V. Senkova, D.B. Miracle, C.F. Woodward, J. Alloys Compd. 509 (2011) 5043-6048.

[6] Y. Zhang, Y.J. Zhou, J.P. Lin, G. L. Chen, and P.K. Liaw, Adv. Eng. Mater. 10 (2008) 534-538.

[7] S.J. Zinkle, and L.L. Snead, Annu. Rev. Mater. Res. 44 (2014) 214-267.

[8] N.A.P.K. Kumar, K.J. Leonard, H. Bei, T.S. Byun, Y. Zhang, and S.J. Zinkle, DOE/ER-0313/54 - Volume 54, Fusion Reactor Materials Program, June 30, 2013.

[9] T. Nagase, P.D. Rack, J.H. Noh, T. Egami, Intermetallics 59 (2015) 32-42.

[10] S.W. Kao, J.W. Yeh, T.S. Chin, J. Phys.: Condens. Mat. 20 (2008) 145214, (7pp)

[11] M.F. del Grosso G. Bozzolo, H.O. Mosca, Physica B 407 (2012) 3285-3287.

[12] S. Wang, and H. Ye, Adv. Mat. Res. 338 (2011) 380-383.

[13] J.C. Duda, T.S. English, D.A. Jordan, P.M. Norris, and
W.A. Soffa, J.Phys.: Condens. Mat. 23 (2011) 205401, (9pp).

[14] A.J.H. McGaughey, M.I. Hussein, E.S. Landry, M. Kaviany, and G.M. Hulbert, Phys. Rev. B 74 (2006) 104304, (9pp).

[15] T. Ikeshoji and B. Hafskjold, Mol. Phys. 81 (1994) 251261.

[16] W.G. Hoover, Computational Statistical Mechanics (Elsevier: Amsterdam, 1991)

[17] G.D. Samolyuk, S.I. Golubov, Y.N. Osetsky, R.E. Stoller, J. Nucl. Mater. 418 (2011) 174-181.

[18] S.J. Plimpton and A.P. Thompson, J. Comp. Phys. 117 (1995) 1-19.

[19] G. Bonny, N. Castin, and D. Terentyev, Model. Simul. Mater. Sci. Eng. 21 (2013) 085004 (15pp).

[20] Y. Mishin, Acta Mater. 52 (2004) 1451-1467.

[21] G.P. Purja Pun, and Y. Mishin, Phys. Rev. B 86 (2012) $134116,(12 \mathrm{pp})$

[22] A. Alam and A. Mookerjee, J of Phys.: Condens. Mat. 18 (2006) 4589-4609.

[23] Thermal Conductivity: Theory, Properties, and Applications (Physics of solids and liquids). Edited by: T.M. Tritt, Clemson University, Clemson, South Carolina, Kluwer Academic/Plenum Publishers, New York, Boston, Dordrecht, London, Moscow, 2004. 